\title{
BMJ Open Is congruent movement training more effective than standard visual scanning therapy to ameliorate symptoms of visuospatial neglect? Study protocol of a randomised control trial
}

\author{
Joris A Elshout, ${ }^{1}$ Tanja C W Nijboer, ${ }^{1,2}$ Stefan Van der Stigchel ${ }^{1}$
}

To cite: Elshout JA,

Nijboer TCW, Van der Stigchel S. Is congruent movement training more effective than standard visual scanning therapy to ameliorate symptoms of visuospatial neglect? Study protocol of a randomised control trial. BMJ Open 2019;9:e031884. doi:10.1136/ bmjopen-2019-031884

- Prepublication history for this paper is available online. To view these files, please visit the journal online (http://dx.doi. org/10.1136/bmjopen-2019031884).

Received 23 May 2019 Revised 17 September 2019 Accepted 23 September 2019

Check for updates

(c) Author(s) (or their employer(s)) 2019. Re-use permitted under CC BY-NC. No commercial re-use. See rights and permissions. Published by BMJ.

${ }^{1}$ Experimental Psychology, Utrecht University, Utrecht, The Netherlands

${ }^{2}$ Center of Excellence for Rehabilitation Medicine, Brain Center Rudolf Magnus, University Medical Center Utrecht, Utrecht University and De Hoogstraat Rehabilitation, Utrecht, The Netherlands

Correspondence to

Joris A Elshout;

j.a.elshout@uu.nl

\section{ABSTRACT}

Introduction Approximately $30 \%$ of all patients with stroke show visuospatial neglect (VSN). Currently, visual scanning therapy (VST) is applied in clinical settings to attenuate neglect symptoms. VST builds on the premise that eye movements to the affected hemifield lead to a concurrent shift of visual attention. Congruent movements with different effectors of the motor system, for example, eye and hand, can produce an even larger boost of attention compared with a single effector. This congruency principle may produce a powerful bias in the motor system, which may counteract the pathological biases in the attentional system of neglect patients. Therefore, an intervention with congruent eye and hand movements may result in greater attenuation of neglect compared with an intervention with single eye movements as applied in standard VST. The current randomised controlled trial will investigate the beneficial effects of this updated version of VST by comparing changes in performance on standard neuropsychological neglect tasks and severity of neglect in activities of daily living.

Methods and analysis Thirty VSN patients in the subacute phase poststroke onset will be randomly assigned to one of two groups: congruent eye and hand movement training (experimental group) versus standard VST (control group). Each patient will receive 10 sessions of training, $30 \mathrm{~min}$ each, within 2 weeks. Performance on standard neuropsychological neglect tasks, a visual discrimination task, severity of neglect in ADL and eye movement characteristics before and after intervention will be compared for and between both groups.

Ethics and dissemination This study has been approved by the ethical committee of the University Medical Centre Utrecht. All subjects will participate voluntarily and will give written informed consent. Results of this study will be published in peer-reviewed scientific journals and presented at international conferences.

Trial registration number NTR7005

\section{INTRODUCTION}

Visuospatial neglect (VSN) is defined as the failure to report, respond to or orient to stimuli presented in the contralesional visual field in the absence of motor or sensory
Strengths and limitations of this study

- In this trial, we study whether a new experimental training is more effective than standard visual scanning therapy to ameliorate symptoms of visuospatial neglect.

- During the experimental training, patients will make congruent eye and hand movements, which has been shown to increase attentional shifts compared with eye movements only in healthy controls.

- Data will be compared with patient (without neglect) and healthy control groups.

- As the subacute patients receive our training parallel with their standard rehabilitation programme, spontaneous neurobiological recovery may interfere with (both) training schedules.

- There will be no long-term follow-up measurement, but we will look at short-term effects.

defects. ${ }^{1}$ The core of this disorder reflects lateralised impairments of attention: there is a strong bias to allocate attention to the ipsilesional visual field, whereas little attention is allocated to the contralesional visual field..$^{2-4}$ Patients with neglect need more help in daily life activities (ADL) compared with patients without neglect ${ }^{56}$ and are less likely to being discharged home after admission to rehabilitation. ${ }^{7}$ While some spontaneous recovery may occur, about $40 \%$ of all VSN patients still show symptoms of neglect 1 year poststroke. ${ }^{8}$ With a high incidence after stroke $(\sim 30 \%)$ and a slow and more attenuated recovery pattern, it is important to develop new and more effective treatments for neglect.

Visual scanning therapy (VST) is currently the most frequently used clinical treatment to attenuate symptoms of VSN. Patients are trained to make systematic eye movements to their affected hemispace. An important assumption for the efficacy of this treatment 
is a concurrent shift of attention coupled to the eye movement. According to the premotor theory of attention (PMT), attention and the motor system are tightly linked. PMT holds that planning a movement is both necessary and sufficient for attentional orienting. ${ }^{9}$ Planning an eye movement to a spatial location results in a shift of attention to that location before the eye movement is executed. This shift is known as a 'presaccadic' shift of attention. ${ }^{10}$ The 'motor system' is not a single system, however, but a collection of different effector systems, such as the eye movement system and the hand movement system. Indeed, planning a hand movement (without moving the eyes) is also preceded by a shift of attention to that location resulting in a 'prepointing' shift of attention. Each effector system might therefore contribute differently and potentially independently to attentional orienting. ${ }^{11}$ Jonikaitis and Deubel investigated this hypothesis and confirmed that both effector systems (hand movements and eye movements) can independently produce a shift of attention. ${ }^{12}$ Importantly, they showed that planning two movements at the same time to the same spatial location (congruent eye-hand movements) results in an additional boost of attention to that location compared with planning a movement with a single effector. These results in healthy controls suggest new approaches to treatment for patients with asymmetric attentional deficits such as VSN. Congruence between different effector systems should produce a powerful bias in the motor system which can counteract pathological biases in the attentional system. Training patients to adopt this principle of congruence outside of the laboratory might result in beneficial effects during various activities in daily life.

Therefore, the current study will evaluate the surplus value of an experimental congruent movement training (CMT: simultaneous eye and hand movements to the same location in the affected hemifield). Results will be compared with a control training with single eye movements, that is, VST. If congruent movement training appears to be more effective than VST in the reduction of VSN symptoms, it might be implemented in the clinic.

\section{METHODS AND ANALYSIS \\ Design}

A new training approach, CMT, will be compared with standard VST in this randomised controlled trial. Since patients are admitted to the rehabilitation centre, which aim to discharge patients from the rehabilitation centre as soon as possible, we chose to compare two independent groups (A vs B) rather than a cross-over design (AB$\mathrm{BA}$ ), which will have led to a large dropout. At the time of the neuropsychological screening to assess whether a patient has VSN, it is still unknown whether a patient can be included in the study and which treatment he/she will receive if he/she can be included. After inclusion, in chronological order, VSN patients will be randomly assigned to one of two groups, based on a predetermined list generated using Matlab, that has paired one of the

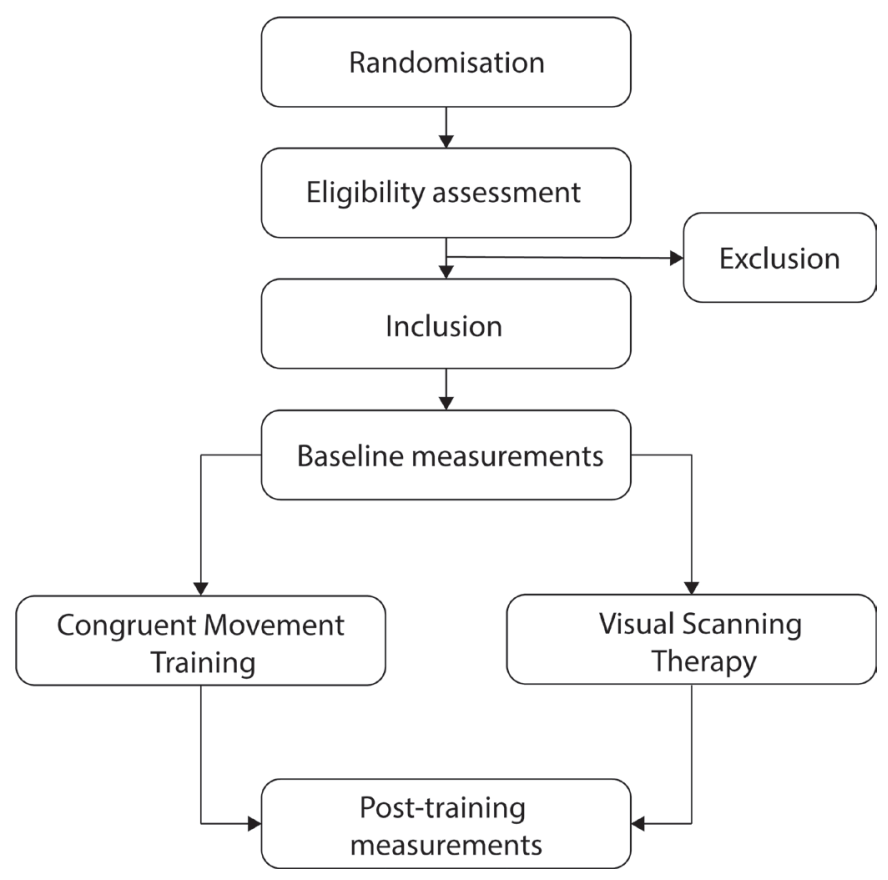

Figure 1 Study procedure.

training variants to a participant number. Patients are not explicitly informed about the nature of their treatment (ie, whether they receive the experimental CMT or control VST treatment). The nurses who assess the Catherine Bergego Scale (CBS) are blinded to training variant. The researchers who administer the training and the tests are not blinded, since they have to explain the training and tests. To minimise any (unintended) bias, the same task instructions are read aloud for each patient before each test. All data are collected and saved automatically by the computer program so that no changes can be made after task completion. Outcome measures of the group of VSN patients who receive CMT $(n=15)$ will be compared with the group of VSN patients who are trained with VST $(n=15)$. All patients will receive 10 sessions of training, $30 \mathrm{~min}$ per session, parallel to their standard rehabilitation programme.

Baseline performance on all tasks will be collected for each VSN patient on two separate days prior to training and compared with the performance after training (figure 1).

\section{Baseline performances}

Since we also include new (secondary) outcome measures and tests (eye tracking, cookie theft picture for free exploration, visual discrimination task, virtual reality), we need to compare the data of the neglect patients to stroke patients without neglect and healthy control to study whether baseline performance (1) deviates from normal range (2) is stroke or neglect specific. In addition, the control data allow us to examine whether performance after training change to values that can be considered as normal on these tasks.

Therefore, only baseline measurements will be collected for a patient control group and healthy control 
group, and these two groups will not receive any training (table 1). Performance on all outcome measures of both VSN intervention groups will be compared with the performance of a patient control group (stroke patients without VSN, $\mathrm{n}=15$ ) and age-matched healthy controls $(\mathrm{n}=15)$.

\section{Subjects}

Patients will be recruited in De Hoogstraat Rehabilitation and De Parkgraaf Rehabilitation between May 2018 and January 2020. VSN is assessed during a neuropsychological screening which is 'care as usual' for all patients admitted to De Hoogstraat and De Parkgraaf Rehabilitation Centre. VSN patients are included (in chronological order) if they (1) are clinical diagnosed with symptomatic stroke (left or right ischaemic or intracerebral haemorrhagic lesion), (2) show signs of VSN based on one of the neuropsychological VSN tests (shape cancellation, line bisection, Catherine Bergego Scale), (3) are between 18 and 85 years of age, (4) have sufficient comprehension and communication, (5) have sufficient motivation to participate and (6) give written informed consent.

The patient control group will have no VSN based on the neuropsychological tests. Healthy control subjects will be included if they have (1) no history of neurological disorders, (2) are between 18 and 85 years of age, (3) sufficient comprehension and communication, (4) sufficient motivation to participate and (5) written informed consent. Exclusion criteria for these groups are (1) interfering disorders, (2) expected discharge $<4$ weeks and (3) mentally or physically unable to participate. The neuropsychologist and rehabilitation physician are consulted regarding the exclusion criteria.

\section{Congruent movement training (experimental treatment)}

CMT is a new approach for treatment of VSN. Patients are instructed to make simultaneous eye and hand movements to a location in their affected hemifield. This is accomplished by performing a game-like task on a computer with touchscreen (figure 2, left pane). The pointing movements are made with the contralesional hand (the ipsilesional hand in case of contralesional paresis). Patients have to match a coloured grid (template grid) that is shown at the top corner of the screen in their affected hemifield. An empty grid is placed beneath the coloured grid (target grid). The patient has to press a button in the ipsilesional hemifield to replace this button with a new coloured item. After appearance of the coloured item in the ipsilesional hemifield, the template grid will flash to attract attention. Subsequently, the patient has to point at a location on the empty grid where he/she wants the coloured item to be placed. The coloured item in the ipsilesional hemifield then will move to the location in the empty grid and the button will appear again. A new coloured item will appear at the ipsilesional field after pressing the button. This task requires repetitive pointing from the ipsilesional side of the screen to specific locations in the affected hemifield and needs to be accompanied by eye movements to the same locations to accurately place the coloured item in the empty grid. Different variations of the task will be available, for example, where a template grid of different shapes (squares, circles and triangles) need to be matched. Patients receive sessions of $30 \mathrm{~min}$, each working day, during 2 weeks.

\section{Visual scanning therapy (control treatment)}

Patients are instructed to make eye movements to the affected hemifield to detect a specific stimulus. Importantly, no pointing movements will be made during this training. To make this task comparable to CMT, two coloured grids (figure 2, right pane) are shown: one at the ipsilesional side (the template grid) and one located in the affected hemifield of the patient (the target grid). The same grids and locations for the target grid are used as those used during CMT. The target grid will flash at random timings to attract attention. The patient has to report verbally if and how many elements of the target grid are different from the template grid. The experimenter will note the response. Different variations of the task will be available (eg, match forms). Patients receive sessions of $30 \mathrm{~min}$, each working day, during 2 weeks.

\section{Study parameters}

Demographical and stroke-related parameters

The demographic factors age, gender, handedness will be registered at baseline. Also, type of stroke, hemisphere, date of stroke, stroke history (recurrent/first), Barthel Index ${ }^{13}$ Motricity Index, ${ }^{14}{ }^{15}$ Montreal Cognitive Assessment ${ }^{16}$ and Stichting Afasie Nederland test ${ }^{17}$ will be registered at baseline.

\section{Main study parameters}

Changes in performance (post-training-baseline) on standard neuropsychological VSN tests, that is, the shape cancellation (number of omissions) and line bisection task (deviation from centre) will be calculated. Also, a visual discrimination task will be used to study the presaccadic shift of attention ${ }^{18}$ before and after training. In this 2-alternative forced choice experimental task, an ' $\mathrm{E}$ ' or inverted ' $E$ ' is presented presaccadically in the left or right hemifield and needs to be reported. Performance (\% correct responses) is suggested to reflect the amount of attention directed to the location of the eye movement and will be calculated per hemifield location. Finally, the CBS, administered by the nurse, will be used to assess changes in ADL. The CBS is an observation list designed to assess the presence and severity of VSN in 10 daily activities. ${ }^{19} 20$ This includes motor components of VSN. For all four primary outcomes, beneficial effects after CMT will be compared with effects after standard VST.

\section{Secondary study outcomes}

To study visual exploration patterns, eye movements will be recorded during an image exploration task. Patients are instructed tolook at and describe the 'Cookie theft' picture. ${ }^{21}$ The number of reported items, search strategies ${ }^{22} 23$ and 


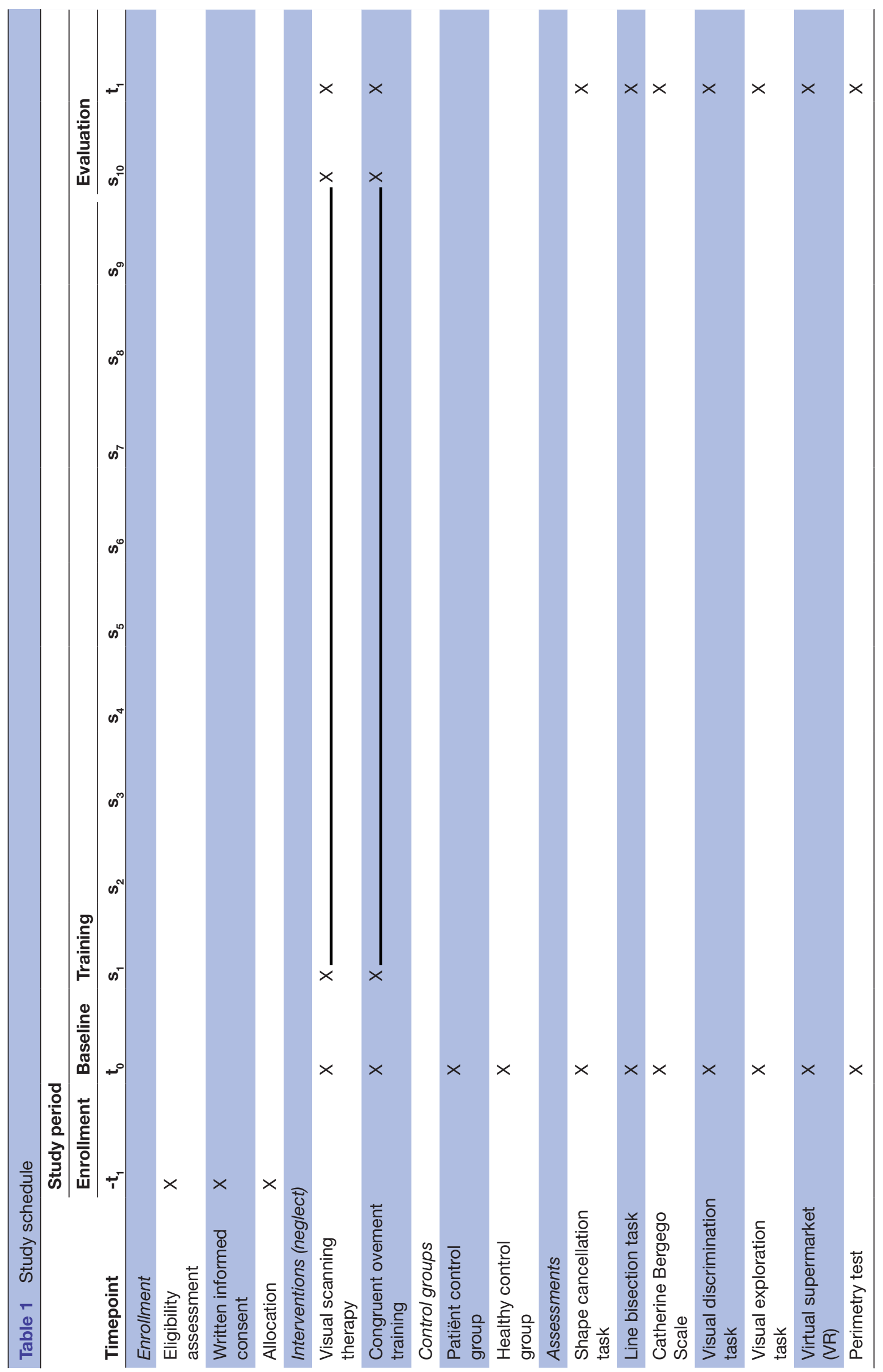




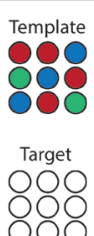

Figure 2 Training paradigm. Left pane: congruent movement training. The template is located at the top or at the bottom of the affected hemifield and the target grid accordingly (most peripheral target of grid $\sim 25^{\circ}$ from fixation). After pressing the button that is placed in the ipsilesional hemifield (indicated by dashed rectangle), a coloured item will appear which needs to be placed at the correct location inside the target grid to match the template grid. Right pane: visual scanning therapy. The target is located at the top or at the bottom (indicated by dashed grid) of the affected hemifield. To report how many items are different, eye movements need to be made between the ipsilesional template grid and the contralesional target grid. Note, this figure is an example for left-sided visuospatial neglect.

the eye movement behaviour (eg, number of fixations) will be obtained.

To study behaviour in a more dynamic situation, patients will perform a shopping task in a virtual supermarket. This supermarket will be administered with an HTC Vive head-mounted display. Eye movements are recorded with build-in eye trackers. With use of the consoles, the patient is able to navigate within the supermarket. Sound is presented through regular headphones. The patients have to purchase a list of products (shopping list shown prior to start) and be alert to announcements via the headphones. The assignment is to find the products on the shopping list before the supermarket closes and walk by the check-out counters to complete the task. Outcomes are number of products found, distance covered, eye movement patterns while searching for products or during navigation.

Also, performance during training (correct responses, time needed to complete trial, number of pointing movements and eye movement characteristics) will be evaluated.

Finally, a computerised visual field test will be used to screen for visual field defects.

For all secondary outcomes, beneficial effects after CMT will be compared with effects after standard VST.

\section{Statistical analyses and sample size calculation}

Repeated measures analyses (analysis of covariance (ANCOVA)) will be performed, with session (baseline post-training) as within-subject variable and treatment (CMT, VST) as between-subject variable. Level of education (Verhage score) will be added as covariate. Performance on all primary and secondary outcome measures will be compared. Patients are randomly assigned to one of the two groups, so we expect that both groups will be comparable in terms of severity of neglect. A difference in severity of neglect between the two groups will be based on chance. However, if there is a difference in severity of neglect, we will correct for that to address our main research question.
In addition, we will conduct additional analysis to relate severity of neglect with training potential in general.

To explore the performance on the new outcome measures (eye tracking data: number of fixations, fixation duration, direction of first saccade) and tasks (cookie theft and virtual supermarket), we compare baseline performances of the neglect patients to the patient and healthy control groups using parametric t-tests or non-parametric tests in case of non-normal distributed data. If these data are deviating from the control groups, we conduct additional analyses, similar as performed for the primary outcome measures (repeated measures analyses (ANCOVA), with session (baseline post-training) as within-subject variable and treatment (CMT, VST) as between-subject variable) to test whether these new outcome measures improve more during CMT than VST training. The false discovery rate approach will be used to correct for multiple comparisons.

We performed an a priori power analysis (G-Power V.3.1) to calculate the sample size. Effect sizes reported in literature vary considerably among studies, ranging from 0 to 2.84, with the highest effect size for combined training methods. A study by Polanowska $e t a l^{24}$ compared somatosensory stimulation +visual scanning training to visual scanning training alone (mean improvement experimental group=58.4 (20.6) vs control group=17.35 (30.3)) showing an effect size of 1.58. Another study by Schroder et al $2008^{25}$ treated neglect patients with Transcutaneous Electrical Nerve Stimulation (TENS) or Optokinetic Stimulation (OKS) compared with a control group that received VST. This study reported an effect size of 0.83 (TENS) and 1.56 (OKS) (mean improvement experimental group TENS=1298 (1.23), experimental group OKS=1938 (0.89) vs control group $=0264(1.25)$ ).

While both study designs and training paradigms are very comparable to our design and training, their amount of training was a bit higher. Therefore, we are more conservative and chose a Cohen's $\mathrm{D}$ of 1 . A power analysis with power set to 0.8 and alpha set to 0.05 estimated the sample size at 14 patients per group (28 in total) for sufficient statistical power.

\section{Patient and public involvement}

Feedback of the patients about the training itself will be used to make the training more appealing, without changing the integrity of the design. Patients and public will not be directly involved in the study design and conducting the study. After study completion, all patients will receive an overview of the main study results.

\section{ETHICS AND DISSEMINATION}

All subjects will participate voluntarily and will give written informed consent after all information about the study protocol is provided via the information letter and by the researchers.

The results of this study will be published in peerreviewed scientific journals and presented at international conferences. 


\section{DISCUSSION}

VST is widely used to ameliorate symptoms of visuospatial VSN. Still, the efficacy of VST is variable and not all VSN patients benefit from this training. ${ }^{26}$ Therefore, the development of new and more effective treatments is important. Recent studies on congruent motor movements in healthy subjects suggest new approaches to treatment; the more effectors of the motor system that agree on the action endpoint, the more attention is allocated to that location. We translated this principle by updating standard VST. A training with congruent eye and hand movements should increase attention to the affected hemifield to a larger extent than a training with single eye movements, as applied during VST. This relatively simple update of standard VST can easily be implemented if shown to be more effective.

We aim to investigate the surplus value of congruent movement training in a randomised design and collect a range of different outcome measures, including eye movement data during all tasks. Eye movement data in clinical tasks may provide additional (objective) insights in underlying problems after stroke, for example, the distribution of visual attention.

A potential pitfall of the study might be that patients receive our training parallel with their standard rehabilitation programme. As patients are in the subacute phase poststroke onset, we expect that almost all patients will show some progress due to neurobiological recovery. ${ }^{827} 28$ A second pitfall might be the relative low amount of training hours. We chose to study the intervention in a rehabilitation centre, with other therapies, in the subacute phase poststroke onset, since this is the setting in which the intervention will be implemented if shown to be more effective than standard VST.

Contributors JAE, TCWN and SVdS contributed to the conception and experimental design of the study. JAE participate in collecting the data and coordination of the study. All authors were involved in writing the manuscript and approved the final version.

Funding This work was supported by the Netherlands Organisation for Scientific Research (NW0; 464-15-112).

Competing interests None declared.

Patient consent for publication Not required.

Ethics approval This study has been approved by the ethical committee of the University Medical Centre Utrecht (NL64626.041.18)

Provenance and peer review Not commissioned; externally peer reviewed.

Open access This is an open access article distributed in accordance with the Creative Commons Attribution Non Commercial (CC BY-NC 4.0) license, which permits others to distribute, remix, adapt, build upon this work non-commercially, and license their derivative works on different terms, provided the original work is properly cited, appropriate credit is given, any changes made indicated, and the use is non-commercial. See: http://creativecommons.org/licenses/by-nc/4.0/.

\section{REFERENCES}

1 Heilman KM, Valenstein E. Mechanisms underlying hemispatial neglect. Ann Neurol 1979;5:166-70.
2 Kinsbourne M. Mechanisms of unilateral neglect. In: Jeannerod M, ed. Neurophysiological and neuropsychological aspects of spatial neglect. Amsterdam: Elsevier Science Publishers, 1987: 69-86.

3 Marshall JC, Halligan PW. Does the midsagittal plane play any privileged role in "left" neglect? Cogn Neuropsychol 1989;6:403-22.

4 Posner MI, Walker JA, Friedrich FJ, et al. Effects of parietal injury on covert orienting of attention. J Neurosci 1984;4:1863-74.

5 Nijboer T, van de Port I, Schepers V, et al. Predicting functional outcome after stroke: the influence of neglect on basic activities in daily living. Front Hum Neurosci 2013a;7:1-6.

6 Nijboer TCW, Kollen BJ, Kwakkel G. The impact of recovery of visuospatial neglect on motor recovery of the upper paretic limb after stroke. PLoS One 2014;9:e100584.

7 Nys GMS, van Zandvoort MJE, de Kort PLM, et al. The prognostic value of domain-specific cognitive abilities in acute first-ever stroke. Neurology 2005;64:821-7.

8 Nijboer TCW, Kollen BJ, Kwakkel G. Time course of visuospatial neglect early after stroke: a longitudinal cohort study. Cortex 2013b;49:2021-7.

9 Rizzolatti G, Riggio L, Dascola I, et al. Reorienting attention across the horizontal and vertical meridians: evidence in favor of a premotor theory of attention. Neuropsychologia 1987;25:31-40.

10 Deubel H, Schneider WX. Saccade target selection and object recognition: evidence for a common attentional mechanism. Vision Res 1996;36:1827-37.

11 Baldauf D, Deubel H. Attentional landscapes in reaching and grasping. Vision Res 2010;50:999-1013.

12 Jonikaitis D, Deubel $\mathrm{H}$. Independent allocation of attention to eye and hand targets in coordinated eye-hand movements. Psychol Sci 2011;22:339-47.

13 Collin C, Wade DT, Davies S, et al. The Barthel ADL index: a reliability study. Int Disabil Stud 1988;10:61-3.

14 Collin C, Wade D. Assessing motor impairment after stroke: a pilot reliability study. J Neurol Neurosurg Psychiatry 1990;53:576-9.

15 Kwakkel G, Wagenaar RC, Twisk JWR, et al. Intensity of leg and arm training after primary middle-cerebral-artery stroke: a randomised trial. The Lancet 1999;354:191-6.

16 Nasreddine ZS, Phillips NA, BÃ@dirian ValÃ@rie, et al. The Montreal cognitive assessment, MoCA: a brief screening tool for mild cognitive impairment. J Am Geriatr Soc 2005;53:695-9.

17 Deelman B, Koning-Haanstra M, Liebrand W, et al. Stichting Afasie Nederland-de SAN-test.Lisse. Swets \& Zeitlinger, 1981.

18 Khan AZ, Blangero A, Rossetti Y, et al. Parietal damage dissociates saccade planning from Presaccadic perceptual facilitation. Cerebral Cortex 2009;19:383-7.

19 Azouvi P, Olivier S, de Montety G, et al. Behavioral assessment of unilateral neglect: study of the psychometric properties of the Catherine Bergego scale. Arch Phys Med Rehabil 2003;84:51-7.

20 Ten Brink AF, Nijboer TCW, Van Beekum L, et al. De Nederlandse Catherine Bergego schaal: Een bruikbaar en valide instrument in de CVA zorg. Wetenschappelijk Tijdschrift voor Ergotherapie 2013;6:27-35.

21 Kaplan E, Goodglass H, Weintraub S. Boston naming test. Philadelphia, PA: Lippincott Williams \& Wilkins, 2001.

22 Dalmaijer ES, Van der Stigchel S, Nijboer TCW, et al. CancellationTools: all-in-one software for administration and analysis of cancellation tasks. Behav Res Methods 2015;47:1065-75.

23 Ten Brink AF, Visser-Meily JMA, Nijboer TCW. What does it take to search organized? the cognitive correlates of search organization during cancellation after stroke. J Int Neuropsychol Soc 2018;24:424-36.

24 Polanowska K, Seniów J, Paprot E, et al. Left-Hand somatosensory stimulation combined with visual scanning training in rehabilitation for post-stroke hemineglect: a randomised, double-blind study. Neuropsychol Rehabil 2009;19:364-82.

25 Schröder A, Wist ER, Hömberg V. Tens and optokinetic stimulation in neglect therapy after cerebrovascular accident: a randomized controlled study. Eur J Neurol 2008;15:922-7.

26 Kerkhoff G, Schenk T. Rehabilitation of neglect: an update. Neuropsychologia 2012;50:1072-9.

27 Kwakkel G, Kollen B, Twisk J. Impact of time on improvement of outcome after stroke. Stroke 2006;37:2348-53.

28 Feeney DM, Baron JC, Diaschisis BJC. Diaschisis. Stroke 1986;17:817-30. 